\title{
I/Q Imbalance Problem in SC-FDMA System with DCT and DFT Basis Functions
}

\author{
F. S. Al-kamali, Hefdhallah Sakran, and N. A. Odhah \\ Department of Electronics, Faculty of Engineering and Architecture, Ibb University, Ibb, Yemen
}

Correspondence should be addressed to F. S. Al-kamali; faisalalkamali@yahoo.com

Received 28 August 2014; Revised 8 January 2015; Accepted 10 January 2015

Academic Editor: Ramón M. Rodríguez-Dagnino

Copyright (C) 2015 F. S. Al-kamali et al. This is an open access article distributed under the Creative Commons Attribution License, which permits unrestricted use, distribution, and reproduction in any medium, provided the original work is properly cited.

In-phase/quadrature-phase (I/Q) imbalance is one of the most serious concerns in the practical implementation of the direct conversion receiver architecture. This paper investigates and analyzes the impacts of the I/Q imbalance on the performance of the single-carrier frequency-division multiple access (SC-FDMA) system with different basis functions by computer simulations only. The suitable phase and amplitude of I and Q branches that can be utilized in SC-FDMA system are studied and determined through simulations for different I/Q imbalance scenarios and different subcarrier mapping schemes. Simulation results show that the phase and amplitude of I and Q branches have significant effects on the bit error rate (BER) performance of the SC-FDMA system.

\section{Introduction}

Due to high spectral efficiency and robustness against frequency-selective fading, orthogonal frequency-division multiple access (OFDMA) has been chosen as a downlink transmission technique in 3GPP long-term evaluation (LTE) standards [1]. Well-known drawbacks of the OFDMA system are the high peak-to-average power ratio and sensitivity to carrier frequency offset (CFO), which result in severe performance degradation. Due to these drawbacks, the use of OFDMA in the uplink mobile environments was found to be impractical to establish [2]. Single-carrier frequencydivision multiple access (SC-FDMA) system has been chosen as an alternative technique in the uplink transmission in 3GPP LTE standards due to its low peak-to-average power ratio $[1,3]$. The performance of the SC-FDMA system suffers from the $\mathrm{CFO}$ and its sensitivity is lower than that of the OFDMA system [3]. Besides the CFO, there are other radio frequency impairments in direct conversion receiver (DCR) of these systems, such as dc-offset and in-phase and quadrature-phase $(\mathrm{I} / \mathrm{Q})$ imbalances, inducing further distortion.

In recent years, DCR has drawn a lot of attention due to its low power consumption and low implementation cost. However, some mismatches in DCR can seriously degrade the system performance [4-7]. The I/Q imbalance is due to the amplitude and phase mismatches between the I and Q branch of the local oscillator whereas the CFO is due to the mismatch of carrier frequency at the transmitter and receiver. In [8-10], It is shown that the I/Q imbalance and CFO can cause a serious intercarrier interference and multiple access interference in SC-FDMA systems. In [8], the issue of CFO in SC-FDMA system is investigated for different basis functions: the discrete Fourier transform (DFT) and the discrete cosine transform (DCT). A new joint equalization and CFO for multiple-input multiple-output SC-FDMA system are proposed and investigated in [9].

The issue of the I/Q imbalance problem in multicarrier systems was extensively investigated in the literature [57]. However, to the best of our knowledge, a few papers have only discussed the I/Q imbalance problem in DFT based SC-FDMA (DFT-SC-FDMA) system [10]. Moreover, the issue of the I/Q imbalance problem in DCT based SC-FDMA (DCT-SC-FDMA) system is not studied in the literature. All that motivated us to do the research of this paper.

The main contribution of this work is to study the issue of the I/Q imbalance problem in DFT-SC-FDMA and DCT-SCFDMA systems. The effects of the I/Q imbalance problem are then compensated using an efficient compensation scheme. 


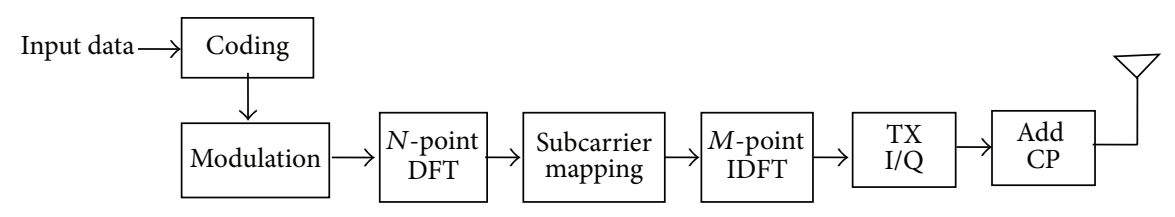

(a) Transmitter of the $u$ th user

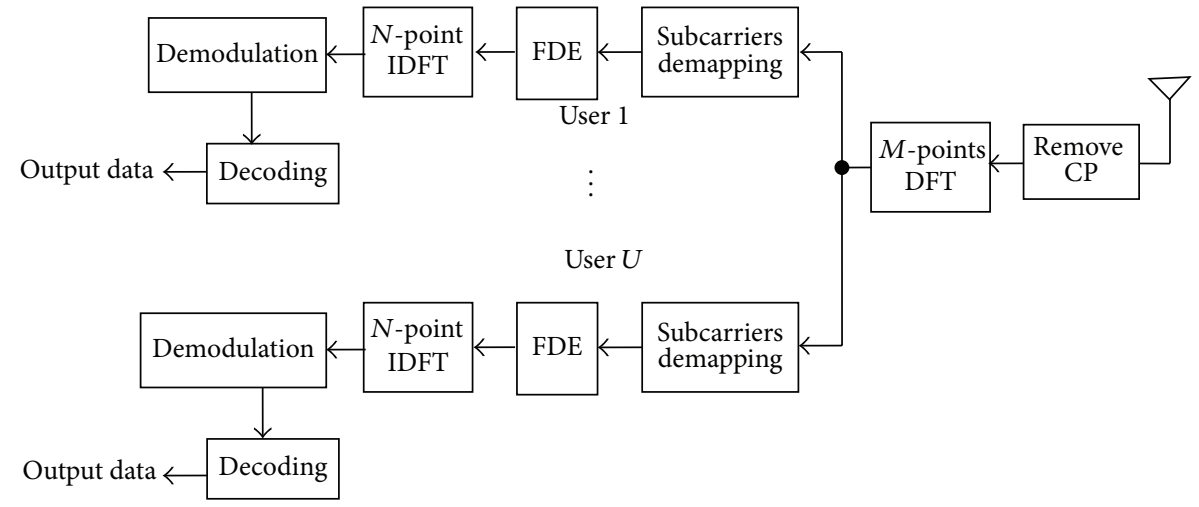

(b) Receiver

FIGURE 1: Structure of the DFT-SC-FDMA system with the TX I/Q imbalance scenario.

The work in this paper is distinct from the previous research works in this area in that we study the effects of the I/Q imbalance problem using a three-dimensional results. On the other hand, the phase and the amplitude of I and Q branches are taken into consideration simultaneously. We investigate different I/Q imbalance scenarios which are the I/Q imbalance in the transmitter (TX I/Q), the I/Q imbalance in the receiver (RX I/Q), and the I/Q imbalances in the transmitter and receiver (TX-RX I/Q). Simulation results show that I/Q imbalance problem significantly degrades the system performance and its effects on the DCT-SC-FDMA system are greater than that on the DFT-SC-FDMA system. Results also show that the proposed compensation scheme can compensate the effects of the I/Q imbalance problem and improve the system performance.

This paper is organized as follows. In Section 2, we develop the DFT-SC-FDMA system model in the presence of the I/Q imbalance problem. The developed I/Q imbalance compensation scheme is discussed in Section 3. In Section 4, we present the simulation results and the paper is concluded in Section 5.

\section{DFT-SC-FDMA System with TX I/Q}

In this section, the DFT-SC-FDMA system model in the presence of I/Q imbalance problem is developed. The extension of this model to the DCT-SC-FDMA system is straightforward. More details about the DCT-SC-FDMA system are found in [3].

Figure 1 depicts the block diagram of the DFT-SC-FDMA system with the TX I/Q imbalance scenario. We consider an uplink DFT-SC-FDMA system employing $U$ users. All users have the same number of subcarriers. The input data is encoded and modulated. The modulated symbols are fed to an $N$-point DFT and then mapped to $M$ subcarriers of assigned subchannels. The signal is then applied to an $M$ point inverse DFT (IDFT) block.

In SC-FDMA system, there are two subcarrier mapping techniques which are the localized mapping and the interleaved mapping. In localized mapping, the DFT outputs are mapped to a subset of consecutive subcarriers, thereby confining them to only a fraction of the system bandwidth. In interleaved mapping, the DFT outputs of the input data are assigned to subcarriers over the entire bandwidth noncontinuously, resulting in zero amplitude for the remaining subcarriers [1]. In this paper, the interleaved DFT-SC-FDMA is denoted by DFT-IFDMA, the localized DFT-SC-FDMA is denoted by DFT-LFDMA, the interleaved DCT-SC-FDMA is denoted by DCT-IFDMA, and the localized DCT-SC-FDMA is denoted by DCT-LFDMA.

The transmitted signal of the $u$ th user $(u=1,2, \ldots, U)$ can be formulated as follows:

$$
\overline{\mathbf{x}}_{u}=\mathbf{F}_{M}^{-1} \mathbf{M}_{u} \mathbf{F}_{N} \widetilde{\mathbf{x}}_{\mathbf{u}}
$$

where $\widetilde{\mathbf{x}}_{u}$ is an $N \times 1$ vector containing the modulated symbols of the $u$ th user. $\mathbf{F}_{N}$ is an $N \times N$ DFT matrix. $\mathbf{F}_{M}^{-1}$ is an $M \times M$ IDFT matrix. $\mathbf{M}_{u}$ is an $M \times N$ matrix describing the subcarriers mapping of the $u$ th user. Also $M=Q \cdot N$, where $Q$ is the maximum number of users that can transmit, simultaneously.

The entries of $\mathbf{M}_{u}$ for both the localized and the interleaved systems are given by $[1,3]$

$$
\begin{gathered}
\mathbf{M}_{u}=\left[\mathbf{0}_{(u-1) N \times N} ; \mathbf{I}_{N} ; \mathbf{0}_{(M-u N) \times N}\right], \\
\mathbf{M}_{u}=\left[\mathbf{0}_{(u-1) \times N} ; \mathbf{u}_{1}^{T} ; \mathbf{0}_{(Q-u) \times N} ; \ldots ; \mathbf{0}_{(u-1) \times N} ; \mathbf{u}_{N}^{T} ; \mathbf{0}_{(Q-u) \times N}\right],
\end{gathered}
$$


where the $\mathbf{I}_{N}$ and $\mathbf{0}_{\mathrm{Q}^{\prime} \times N}$ matrices denote the $N \times N$ identity matrix and the $Q^{\prime} \times N$ all-zero matrix, respectively. $\mathbf{u}_{l}(l=$ $1,2, \ldots, N)$ denotes the unit column vector, of length $N$, with all-zero entries except at $l$. The TX I/Q imbalance problem distorts the ideal transmitted signal as follows:

$$
\mathbf{x}_{\mathbf{u}}=\alpha_{u} \overline{\mathbf{x}}_{u}+\beta_{u} \overline{\mathbf{x}}_{u}^{*},
$$

where $\overline{\mathbf{x}}_{u}^{*}$ is the conjugate of $\overline{\mathbf{x}}_{u}$. The two complex scalars $\alpha_{u}$ and $\beta_{u}$ are given by [9]

$$
\begin{aligned}
& \alpha_{u}=\cos \phi_{u}+j \varepsilon_{u} \sin \phi_{u}, \\
& \beta_{u}=\varepsilon_{u} \cos \phi_{u}-j \sin \phi_{u},
\end{aligned}
$$

where $\varepsilon_{u}$ and $\phi_{u}$ denote the phase and amplitude imbalances between I and Q branches of the transmitted signal of the $u$ th user, respectively. When the matching of I and Q balances is ideal, that is, $\varepsilon_{u}=1$ and $\phi_{u}=0$, then $\alpha_{u}=1$ and $\beta_{u}=0$. At the end of the transmitter, the cyclic prefix (CP) is appended to $\mathbf{x}_{\mathbf{u}}$. The length of the CP must be longer than that of the channel. The channel after removal of the $\mathrm{CP}$ at the receiver can be described by an equivalent $M \times M$ circulant channel matrix $\mathbf{H}_{\mathbf{u}}$, whose first column is given by $\mathbf{h}_{u}=\left[h_{u}(0), h_{u}(1), \ldots, h_{u}(L-1), 0_{1 \times(M-L)}\right] . h_{u}(n)(n=$ $0,1,2, \ldots, L-1)$ is the multipath channel impulse response between the $u$ th user and the base station. $L$ is the length of the channel impulse response.

At the receiver side, the $\mathrm{CP}$ is removed from the received signal and the received signal can be written as follows:

$$
\mathbf{r}=\sum_{u=1}^{U} \mathbf{H}_{u} \mathbf{x}_{u}+\mathbf{n},
$$

where $\mathbf{x}_{u}$ is an $M \times 1$ vector representing the block of the transmitted symbols of the $u$ th user. $\mathbf{n}$ is an $M \times 1$ vector describing the additive noise. It contains independent identically distributed zero-mean additive white Gaussian noise. Since the circulant matrix can be diagonalized by DFT matrix [3], the received signal block after DFT is described as follows:

$$
\mathbf{R}=\sum_{u=1}^{U} \Lambda_{u} \mathbf{F}_{M}\left(\alpha_{u} \overline{\mathbf{x}}_{u}+\beta_{u} \overline{\mathbf{x}}_{u}^{*}\right)+\mathbf{N}
$$

where $\Lambda_{u}$ is an $M \times M$ diagonal matrix containing the DFT of the circulant sequence of $\mathbf{H}_{u}$; that is, $\mathbf{H}_{\mathbf{u}}=\mathbf{F}_{M}^{-1} \boldsymbol{\Lambda}_{u} \mathbf{F}_{M}$ [3]. $\mathbf{N}$ is the DFT of $\mathbf{n}$.

After that, the demapping, the frequency domain equalization (FDE), and the IDFT operations are performed to provide the estimate of the modulated symbols as follows:

$$
\widehat{\widetilde{\mathbf{x}}}_{u}=\mathbf{F}_{N}^{-1} \mathbf{W}_{u} \mathbf{M}_{u}^{T} \mathbf{R}
$$

where $\mathbf{W}_{u}$ is the $N \times N$ FDE matrix of the $u$ th user. $\mathbf{M}_{u}^{T}$ is the $N \times M$ subcarriers demapping matrix of the $u$ th user. It is the transport of the subcarrier mapping matrix.

Finally, the demodulation and the decoding processes are performed.
The RX I/Q imbalance problem distorts the ideal received signal as follows:

$$
\overline{\mathbf{r}}=\alpha_{u} \mathbf{r}+\beta_{u} \mathbf{r}_{u}^{*},
$$

where $\mathbf{r}$ is the received signal after removing the CP.

\section{Proposed RX I/Q Imbalance Compensation Scheme}

One limiting issue in implementing high-speed wireless systems is the impairment associated with analog processing due to component imperfections. I/Q imbalance is a major source of such impairment and it is one of the main practical obstacles in the implementation of DCR. This highlights the need for compensation scheme for IQ imbalance. There have been many reports in the literature on the compensation of the I/Q imbalance in orthogonal frequency-division multiplexing (OFDM) systems [6, 10-12]. Several compensation methods for I/Q imbalance in OFDM systems have been proposed in [6]. In [10] the effects of the I/Q imbalance problem on the performance of the OFDMA and SC-FDMA systems are investigated and compensated. In [11], assuming that the channel frequency response is smooth, a frequency domain estimation method has been proposed to jointly estimate the I/Q imbalance and channel frequency response. I/Q imbalance estimation and compensation in OFDM systems have been studied in [12].

In this paper, the I/Q imbalance compensation scheme in [11] is developed to compensate the effects of the I/Q imbalance in SC-FDMA system. As we will see in the simulation results, the RX I/Q scenario has greater effects on the system performance than that of the TX I/Q scenario and this scenario must be properly compensated at the receiver. Thus, only the RX I/Q compensation will be discussed in this paper. Define the I/Q parameter

$$
\mu=\frac{\beta}{\alpha^{*}} .
$$

Then from (8), we can write the vector $\mu \mathbf{r}$ as follows [11]:

$$
\mu \mathbf{r}=\frac{\mathbf{r}-\mu \mathbf{r}^{*}}{1-|\mu|^{2}} .
$$

Thus if the I/Q parameter $\mu$ is known at the receiver, we can use the above equation to compensate the impact of the RX I/Q scenario.

After that, the signal in (10) is transformed into the frequency domain followed by the demapping, the FDE, the IDFT, the demodulation, and the decoding operations.

\section{Simulation Results}

In this section, the performance of the DFT-SC-FDMA and the DCT-SC-FDMA systems in the presence of I/Q imbalance problem has been evaluated by simulations. Convolutional code with memory 7 and octal generator 
TABLE 1: Simulation parameters.

\begin{tabular}{lc}
\hline Parameter & Description \\
\hline System bandwidth & $5 \mathrm{MHz}$ \\
modulation & QPSK \\
Cyclic prefix & 20 symbols \\
$M$ & 512 \\
$N$ & 128 \\
I/Q imbalance scenarios & TX I/Q, RX I/Q, and TX-RX \\
& I/Q \\
Subcarriers mapping method & DFT-IFDMA, DFT-LFDMA, \\
& DCT-IFDMA, and \\
Channel estimation & DCT-LFDMA \\
Equalization & Perfect \\
\hline
\end{tabular}

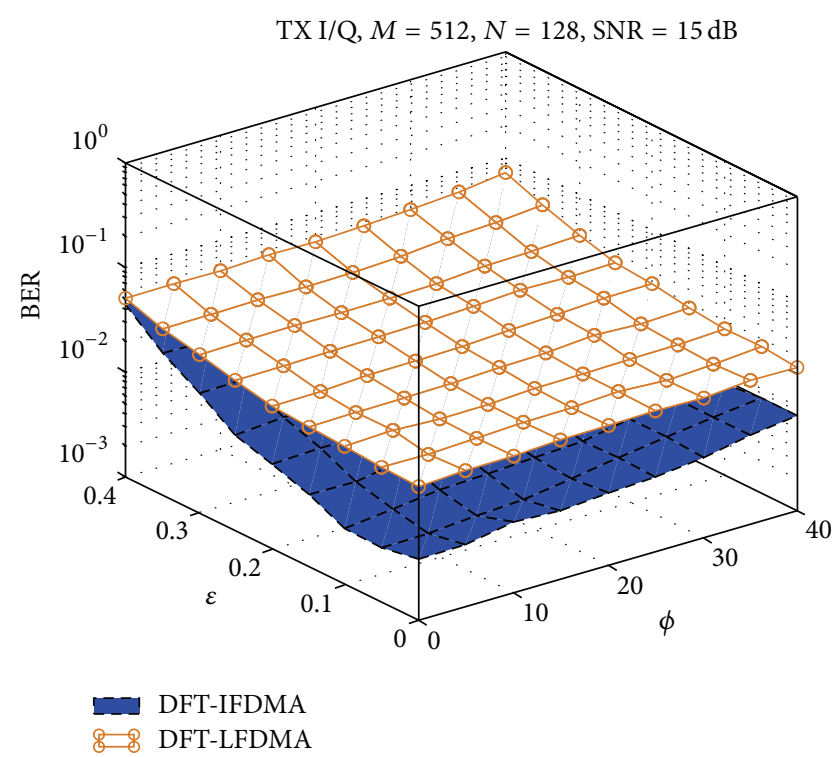

FIgURE 2: Variation of the BER with both $\varepsilon$ and $\phi$ for the DFTIFDMA and the DFT-LFDMA systems when the TX I/Q imbalance scenario is used.

polynomials $(133,171)$ is chosen as channel code. QPSK is used. The channel model used for simulations is vehicular A outdoor channel [13]. The fading was modeled as quasistatic. Simulation parameters are tabulated in Table $1 . \varepsilon$ and $\phi$ are randomly generated for each iteration.

Figures 2 and 3 demonstrate the impact of the TX I/Q imbalance scenario on the performance of the DFTSCFDMA and DCT-SC-FDMA systems with different subcarriers mapping schemes and a SNR $=15 \mathrm{~dB}$. Figures 2 and 3 show the variation of the BER with both $\varepsilon$ and $\phi$ when the TX I/Q imbalance scenario is considered. On the other hand, $\varepsilon$ and $\phi$ are taken into account simultaneously. It is clearly seen that the performance of the two systems deteriorates at high values of $\varepsilon$ and $\phi$, especially with the interleaved subcarrier mapping. From these figures, it is clear that for
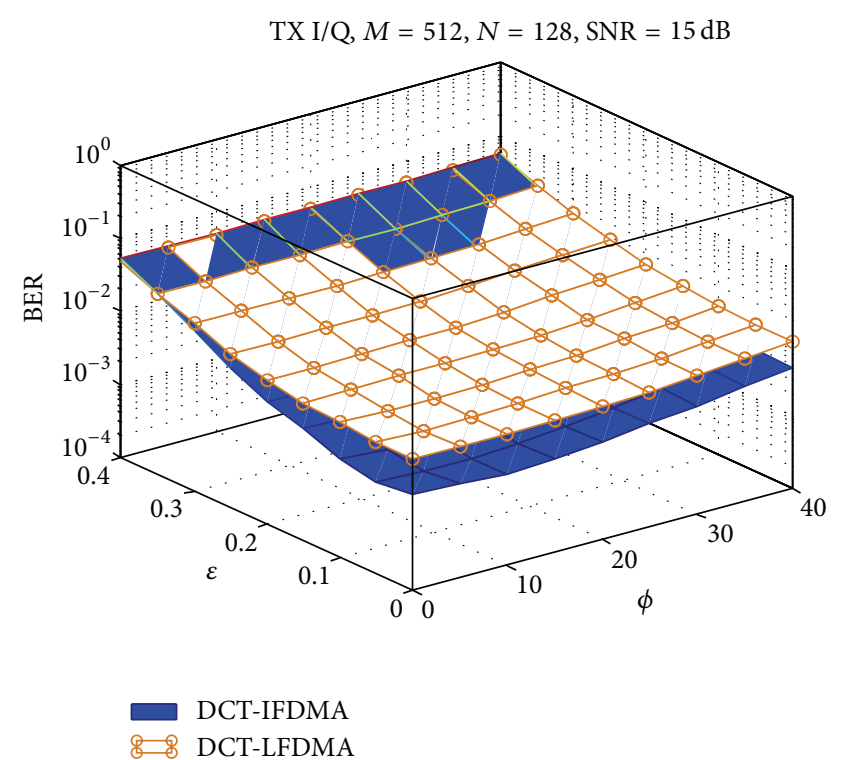

FIGURE 3: Variation of the BER with both $\varepsilon$ and $\phi$ for the DCTIFDMA and the DCT-LFDMA systems when the TX I/Q imbalance scenario is used.

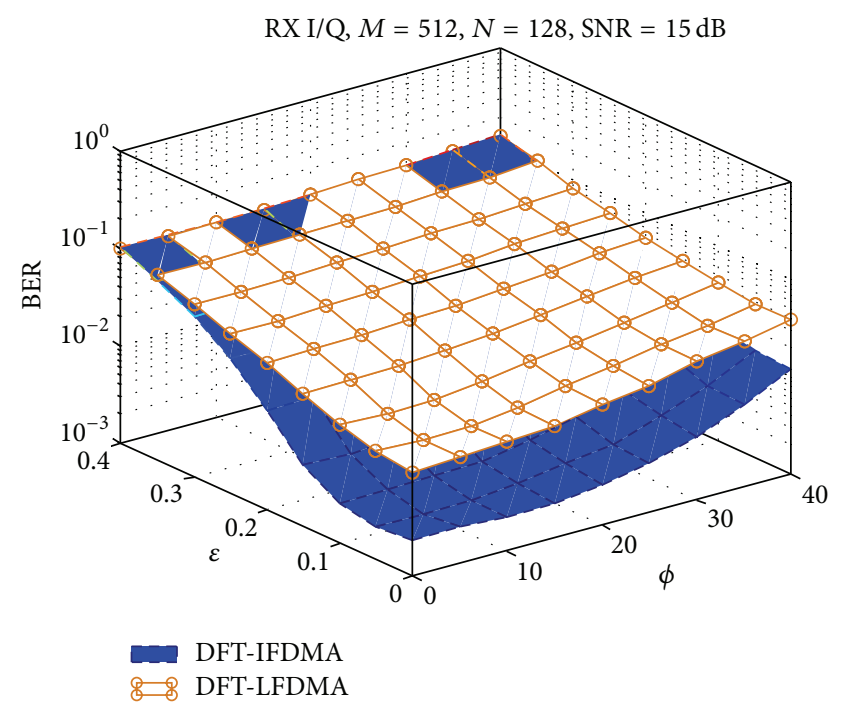

FIGURE 4: Variation of the BER with both $\varepsilon$ and $\phi$ for the DFTIFDMA and the DFT-LFDMA systems when the RX I/Q imbalance scenario is used.

small to moderate values of $\varepsilon$ and $\phi$, the performances of both systems are insensitive to $\varepsilon$ and $\phi$.

Figures 4 and 5 show the variation of the BER with both $\varepsilon$ and $\phi$ when the RX I/Q imbalance scenario is considered. It is shown that the performance of the DFT-SC-FDMA and DCT-SC-FDMA systems seriously deteriorates, especially at high values of $\varepsilon$ and $\phi$. It is also noted that the effects of the RX I/Q imbalance scenario on the DCT-SC-FDMA system are greater than that on the DFT-SC-FDMA system, especially with the interleaved mapping.

From Figures 2 to 5 it is clear that the effects of the RX I/Q imbalance on the performance of the DFT-SC-FDMA and 


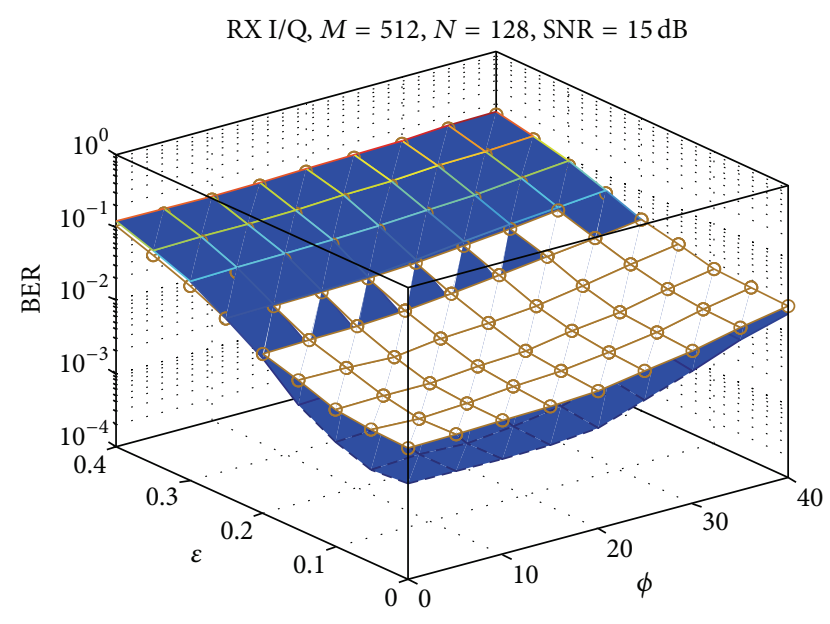

$\square$ DCT-IFDMA

FIGURE 5: Variation of the BER with both $\varepsilon$ and $\phi$ for the DCTIFDMA and the DCT-LFDMA systems when the RX I/Q imbalance scenario is used.

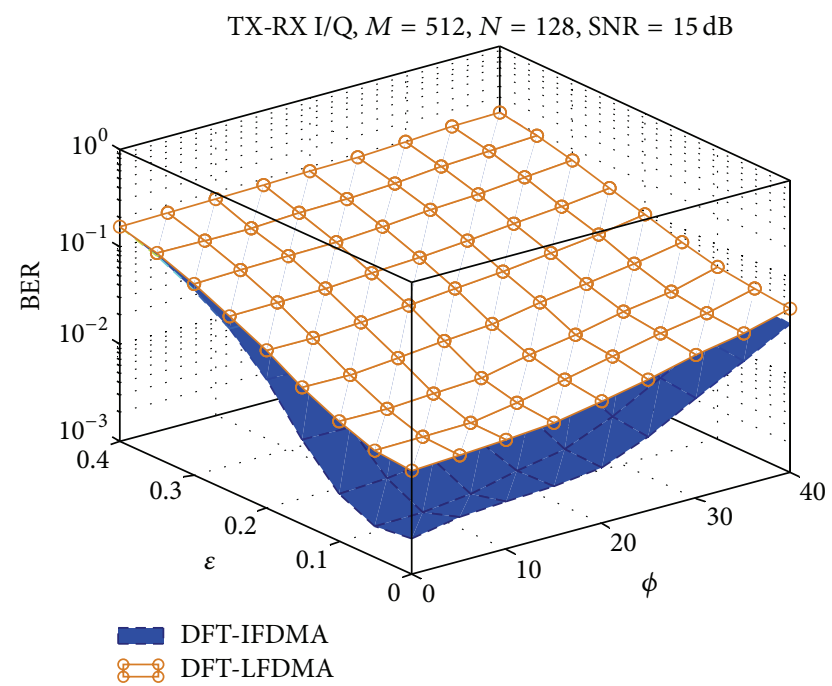

FIGURE 6: Variation of the BER with both $\varepsilon$ and $\phi$ for the DFTIFDMA and the DFT-LFDMA systems when the TX-RX I/Q imbalance scenario is used.

DCT-SC-FDMA system are greater than that of the TX I/Q imbalance, especially at high $\varepsilon$ and $\phi$ values. On the other hand, the larger the values of $\varepsilon$ and $\phi$, the higher the BER and the worse the performance. Also, the lower the values of $\varepsilon$ and $\phi$, the lower the BER and the better the performance. It is also shown that the effects of $\varepsilon$ are greater than that of $\phi$.

Figures 6 and 7 study the effects of the TX-RX I/Q imbalance scenario on the performance of the DFT-SCFDMA and DCT-SC-FDMA systems with different subcarriers mapping schemes and a $\mathrm{SNR}=15 \mathrm{~dB}$. These figures show that the TX-RX I/Q scenario has the highest effects on the system performance of both systems when compared with that of the TX I/Q and RX I/Q scenarios. Figures 3, 5, and

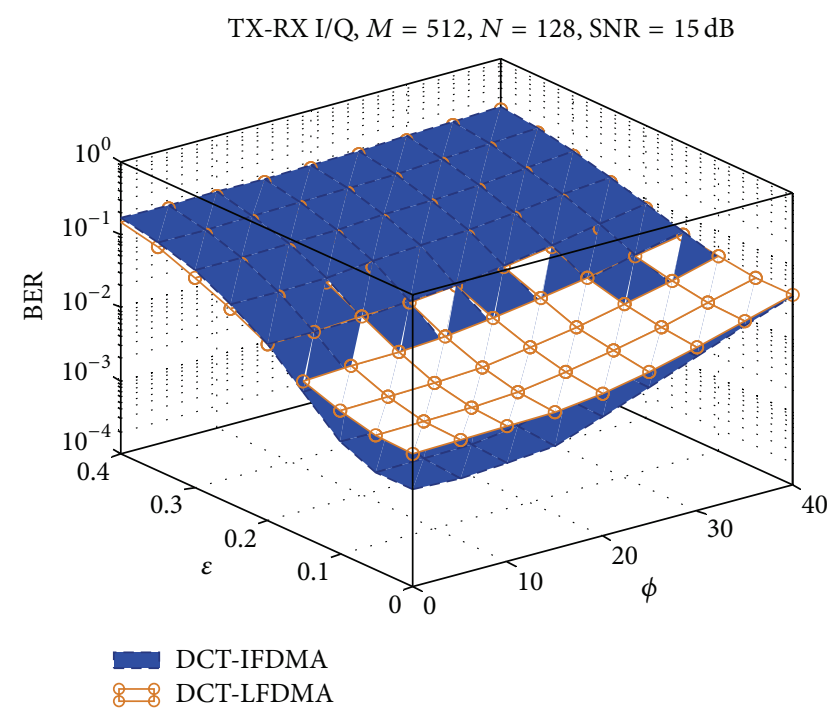

FIgURE 7: Variation of the BER with both $\varepsilon$ and $\phi$ for the DCTIFDMA and the DCT-LFDMA systems when the TX-RX I/Q imbalance scenario is used.

7 indicate that the performance of the DCT-LFDMA is better than that of the DCT-IFDMA at high $\varepsilon$ values, regardless of the $\phi$ values. This is because that the performance of the interleaved system is more sensitive to the multiple access interference than the performance of the localized system [3].

Figures 8 and 9 show the BER performances versus SNR for the DFT-SC-FDMA and DCT-SC-FDMA systems for different I/Q imbalance scenarios. Each $\varepsilon_{u}$ is a random variable with uniform distribution in $[0,0.2]$ and each $\phi_{u}$ is a random variable with uniform distribution in $[-\pi / 8, \pi / 8]$. For the TX I/Q scenario, we can clearly see that the BER performance is better than that of the RX I/Q scenario at high SNR. This is because the interference is dominant at high SNR values. However, the performances of these scenarios are nearly the same at low SNR values, when $\mathrm{SNR}<5$, because the noise is dominant at low SNR region. It is also clear that the performance of the TX-RX scenario is the worst as compared with the other scenarios. Thus, adding an efficient compensation scheme for SC-FDMA systems is very important to compensate the effects of the I/Q imbalance problem.

With a BER of $10^{-3}$, the required SNR values for the DCT-IFDMA and DFT-IFDMA systems without TX I/Q imbalance are $16 \mathrm{~dB}$ and $16.25 \mathrm{~dB}$, respectively, whereas the required SNR for the DCT-IFDMA and DFT-IFDMA systems with TX I/Q imbalance are $20 \mathrm{~dB}$ and $18.75 \mathrm{~dB}$, respectively, which demonstrates a loss of $4 \mathrm{~dB}$ and $2.5 \mathrm{~dB}$ for the DCT-IFDMA and DFT-IFDMA, respectively. This illustrates that the effects of the TX I/Q imbalance on the DCTIFDMA system are greater than that on the DFT-IFDMA system.

Figures 8 and 9 also show the performance of the proposed RX I/Q compensation scheme for the DFT-SCFDMA and DCT-SC-FDMA systems, respectively. It is clearly 

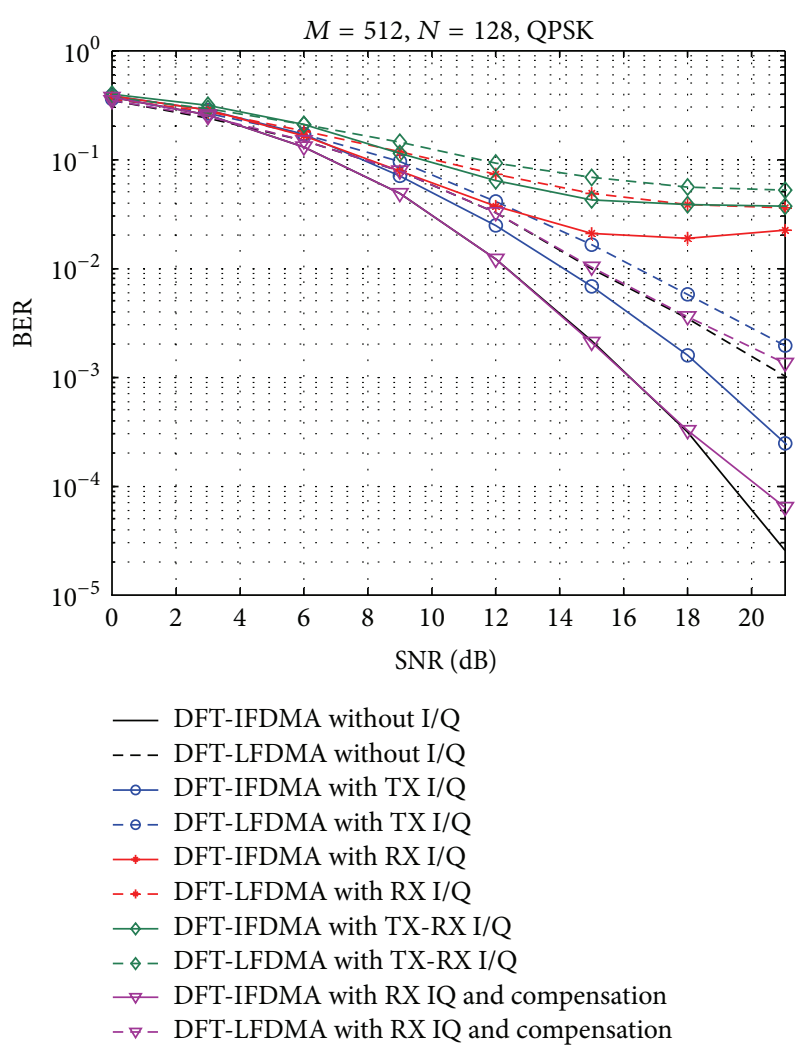

FIGURE 8: BER versus SNR for the DFT-IFDMA and the DFTLFDMA systems with different I/Q imbalance scenarios and RX I/Q imbalance compensation scheme.

seen that the proposed compensation scheme can nearly remove the effects of the RX I/Q imbalance scenario and provide nearly the same performance as that without RX I/Q imbalance.

\section{Conclusions}

In this paper, the issue of the I/Q imbalance problem in the DFT-SC-FDMA and DCT-SC-FDMA systems was studied and the obtained results might be taken into account in a practical design. It was illustrated that the IQ imbalance problem can cause large degradations in the DFT-SC-FDMA and DCT-SC-FDMA receivers. The RX I/Q scenario has significant effects on the performance degradation compared with that of the TX I/Q scenario and these effects depend on the subcarrier mapping used in the system. It was also noted that the effects of the I/Q imbalance problem on the performance of the DC-SC-FDMA system are greater than that on the performance of the DFT-SC-FDMA system, especially for the interleaved mapping scheme. Simulation results also demonstrated that the difference between the BER values in the region with $\varepsilon$ varying from 0 to 0.1 and $\phi$ varying from 1 to $20^{\circ}$ is nearly negligible and the system performance is not sensitive to these parameters in this region. The region of the minimum BER is nearly the same for the DFT-SCFDMA and DCT-SC-FDMA systems with all I/Q scenarios: the TX I/Q, the RX I/Q, and the TX-RX I/Q.
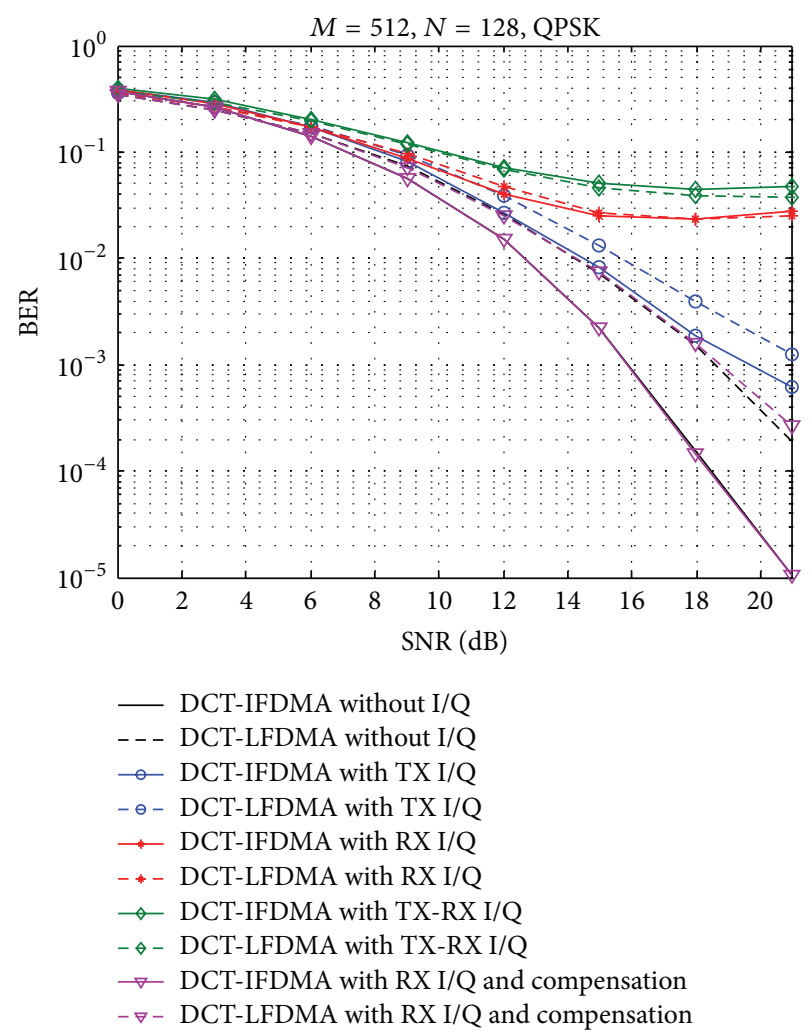

FIGURE 9: BER versus SNR for the DCT-IFDMA and DCT-LFDMA systems with different I/Q imbalance scenarios and RX I/Q imbalance compensation scheme.

Also, an efficient RX I/Q compensation scheme for the DFT-SC-FDMA and DCT-SC-FDMA receivers was developed. It was found that the developed scheme can effectively improve the system performance and reduce the sensitivity of the DFT-SC-FDMA and DCT-SC-FDMA receivers to the RX $\mathrm{I} / \mathrm{Q}$ imbalance problem.

\section{Conflict of Interests}

The authors declare that there is no conflict of interests regarding the publication of this paper.

\section{References}

[1] H. G. Myung and D. J. Goodman, Single Carrier FDMA: A New Air Interface for Long Term Evaluation, John Wiley \& Sons, Chichester, UK, 2008.

[2] B. Farhang-Boroujeny, "Filter bank multicarrier modulation: a waveform candidate for $5 \mathrm{G}$ and beyond," Advances in Electrical Engineering, vol. 2014, Article ID 482805, 25 pages, 2014.

[3] F. E. Abd El-Samie, F. S. Al-kamali, A. Y. Al-Nahari, and M. I. Dessouky, SC-FDMA for Mobile Communications, CRC Press, 2013.

[4] B. Razavi, RF Microelectronics, Prentice-Hall, Englewood Cliffs, NJ, USA, 1998.

[5] C. L. Liu, "Impacts of I/Q imbalance on QPSK-OFDM-QAM detection," IEEE Transactions on Consumer Electronics, vol. 44, no. 3, pp. 984-989, 1998. 
[6] A. Tarighat, R. Bagheri, and A. H. Sayed, "Compensation schemes and performance analysis of IQ imbalances in OFDM receivers," IEEE Transactions on Signal Processing, vol. 53, no. 8, pp. 3257-3268, 2005.

[7] Y.-H. Chung and S.-M. Phoong, "Joint estimation of I/Q imbalance, CFO and channel response for MIMO OFDM systems," IEEE Transactions on Communications, vol. 58, no. 5, pp. 14851492, 2010.

[8] F. S. Al-Kamali, M. I. Dessouky, B. M. Sallam, F. Shawki, and F. E. Abd El-Samie, "Equalization and carrier frequency offsets compensation for the SC-FDMA system," Wireless Personal Communications, vol. 67, no. 2, pp. 113-138, 2012.

[9] F. S. Al-Kamali, M. I. Dessouky, B. M. Sallam, F. Shawki, W. Al-Hanafy, and F. E. A. El-Samie, "Joint low-complexity equalization and carrier frequency offsets compensation scheme for MIMO SC-FDMA systems," IEEE Transactions on Wireless Communications, vol. 11, no. 3, pp. 869-873, 2012.

[10] Y. Yoshida, K. Hayashi, H. Sakai, and W. Bocquet, "Analysis and compensation of transmitter IQ imbalances in OFDMA and SC-FDMA systems," IEEE Transactions on Signal Processing, vol. 57, no. 8, pp. 3119-3129, 2009.

[11] J. Tubbax, B. Côme, L. van der Perre et al., "Compensation of IQ imbalance and phase noise in OFDM systems," IEEE Transactions on Wireless Communications, vol. 4, no. 3, pp. 872877, 2005.

[12] J. Tubbax, A. Fort, L. V. Der Perre et al., "Joint Compensation of IQ imbalance and Frequency Offset in OFDM systems," in Proceedings of the IEEE Global Telecommunications Conference (GLOBECOM '03), pp. 2365-2369, December 2003.

[13] 3rd Generation Partnership Project, "Technical specification group radio access network; user equipment (UE) radio transmission and reception (FDD) (Release 7)," Tech. Rep. 3GPP TS 25.101, Section B.2.2, 2007. 

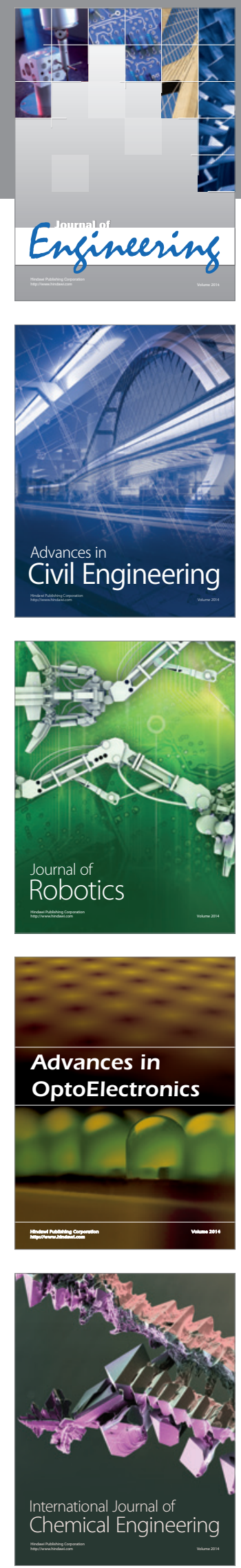

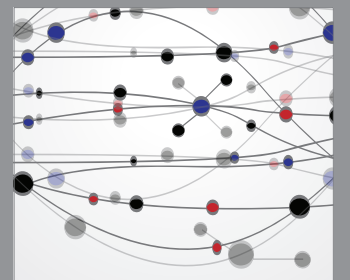

The Scientific World Journal
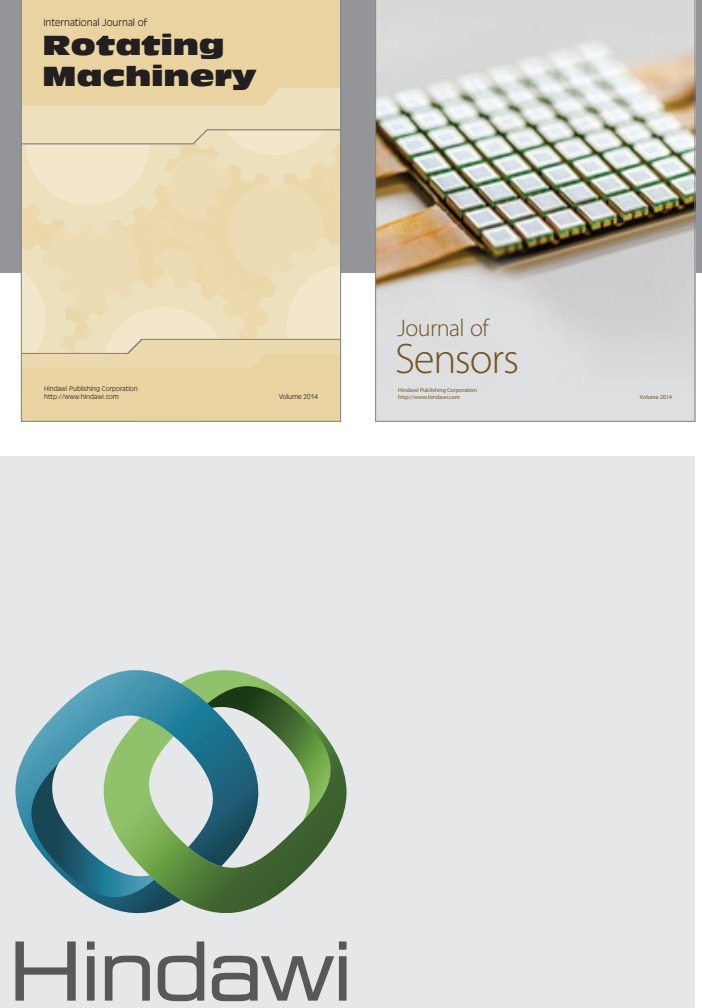

Submit your manuscripts at http://www.hindawi.com
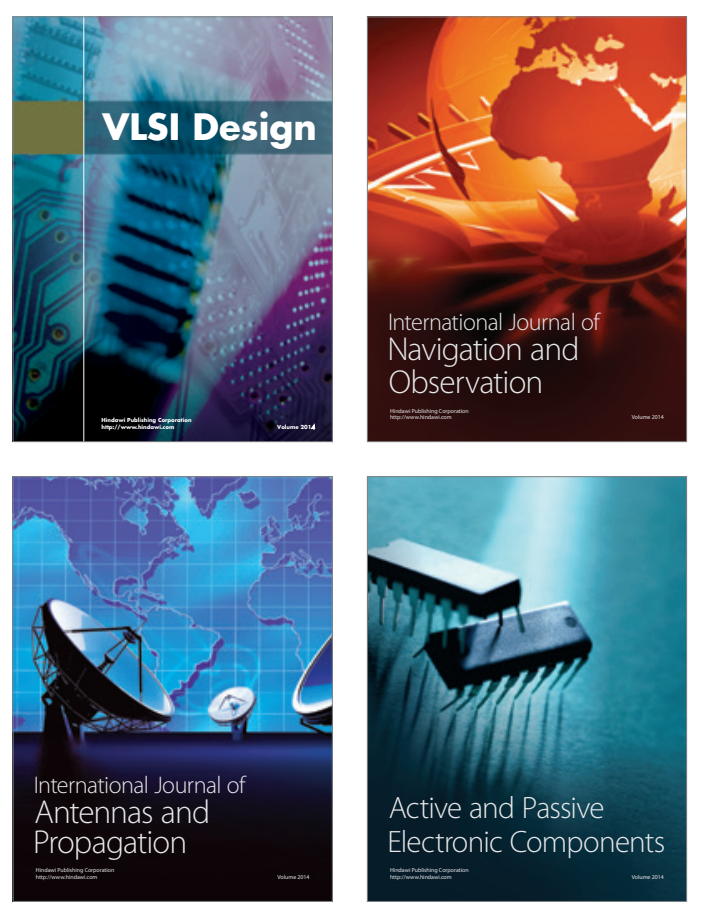
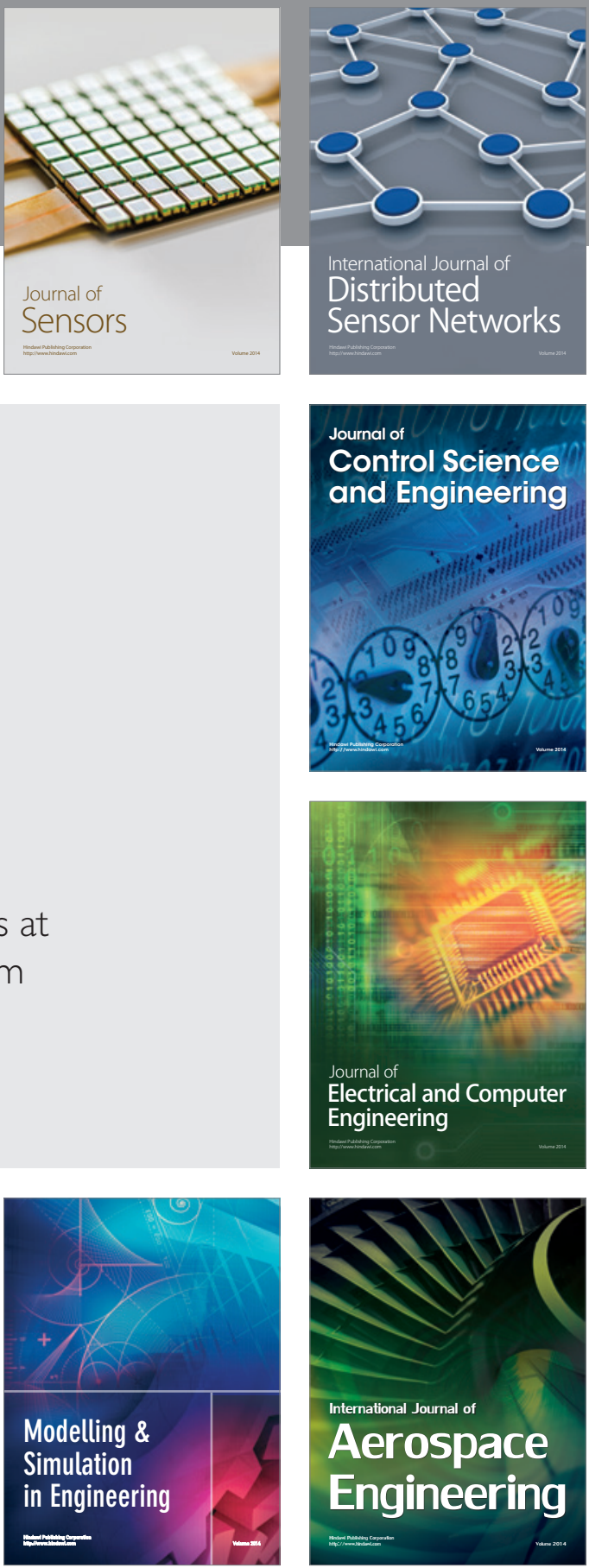

Journal of

Control Science

and Engineering
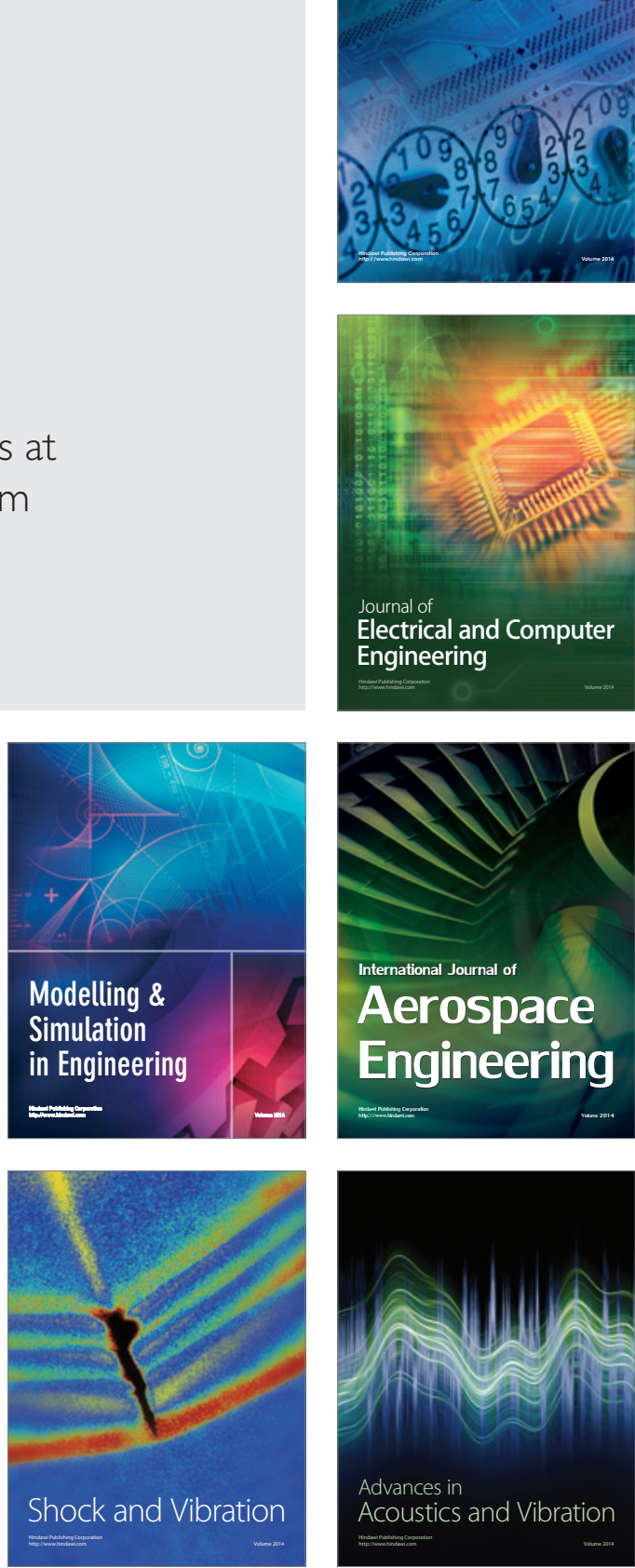